\title{
As Tecnologias Digitais da Informação e Comunicação como um recurso didático no Currículo de Matemática
}

\section{Digital information and communication technologies as a didactic resource in the mathematics curriculum}

\section{Tecnologías digitales de información y comunicación como recurso didáctico en el currículo de matemáticas}

Iaqchan Ryokiti Homa-Agostinho

iaqchan@ulbra.br

Programa de Pós-graduação em Ensino de Ciências e Matemática

Universidade Luterana do Brasil

Canoas, Rio Grande do Sul, Brasil.

Orcid: https://orcid.org/0000-0002-5771-1319
Claudia Lisete Oliveira-Groenwald

claudiag@ulbra.br

Programa de Pós-graduação em Ensino de Ciências e Matemática

Universidade Luterana do Brasil

Canoas, Rio Grande do Sul, Brasil.

Orcid: https://orcid.org/0000-0001-7345-8205

Received: 19/nov/2019・Accepted: 1/mar/2020 • Published: 31/jul/2020

\section{Resumo}

Este artigo apresenta os resultados de pesquisa do projeto Educação Matemática e Tecnologias Digitais, desenvolvida no grupo de pesquisa de Estudos Curriculares de Educação Matemática (GECEM), do Programa de Pós-graduação em Ensino de Ciências e Matemática (PPGECIM), da Universidade Luterana do Brasil (ULBRA), em Canoas, no estado do Rio Grande do Sul, Brasil. Entende-se que as tecnologias alteraram o modo de interação e de pensamento do ser humano em relação ao mundo que o rodeia e neste período de informatização massiva, no qual as atividades têm migrado para o formato digital, a Educação e a Educação Matemática também necessitam se adequar a essa realidade. A pergunta que move as pesquisas ligadas às Tecnologias Digitais do grupo GECEM é: quais as possibilidades didáticas das tecnologias digitais para a Educação Matemática na educação básica e no ensino superior? Levando a análise sobre o potencial pedagógico das tecnologias digitais (computadores, tablets, smartphones, calculadoras) na educação matemática, tanto na educação básica quanto no ensino superior (formação de professores e cursos que utilizam a matemática como suporte na formação profissional). A metodologia utilizada durante a pesquisa foi do tipo exploratória de natureza analítico-descritiva. Foram apresentados um simulador de um braço robótico, um objeto de aprendizagem para visualização da área de triângulos e uma sequência didática com a temática Estatística e Educação Ambiental, cujos resultados, encontrados com a utilização de recursos digitais, foram positivos e demonstram potencial para ser utilizados pelos professores na sala de aula, podendo ser explorados no planejamento didático, tanto na educação básica quanto na formação de professores.

Palavras-chaves: Educação matemática; Educação multimídia; Estratégias Educativas; STEM; Simuladores de Robótica 


\section{Abstract}

This article presents the results of the project entitled Mathematics Education and Digital Technologies, conducted in the research group of Curriculum Studies of Mathematics Education (GECEM), of the Graduate Program in Science and Mathematics Education (PPGECIM), from Universidade Luterana do Brasil (ULBRA), Canoas, State of Rio Grande do Sul, Brazil. It is understood that technologies have changed the way humans interact and think regarding the world around them, and mathematics education needs to adapt to this reality. The problem that motivated the GECEM group to conduct this research on digital technologies was: What are the didactic possibilities of digital technologies for mathematics education in basic and higher education? The paper analyzes the pedagogical potential of digital technologies (computers, tablets, smartphones, calculators) in mathematics education, both in basic and higher education (teacher training and courses that use mathematics as support in professional training). The methodology used during the research was exploratory with an analytical-descriptive approach. For instance, a robotic arm simulator was used as a learning object for the visualization of triangle areas and didactic sequence, for the statistics and environmental education topic. The results of using digital resources have been positive, and such resources have potential to be used by teachers in the classroom; therefore, they should be explored for didactic planning, both in basic education as well as to train teachers.

Keywords: Mathematics education; multimedia teaching; educational strategies; STEM; Robotics Simulators

\section{Resumen}

Este artículo presenta los resultados de la investigación del proyecto Educación Matemática y Tecnologías Digitales, desarrollado en el grupo de investigación de Estudios Curriculares de Educación Matemática (GECEM), del Programa de Posgrado en Enseñanza de Ciencias y Matemáticas (PPGECIM), de la Universidad Luterana del Brasil (ULBRA), en Canoas, provincia del Rio Grande del Sur, Brasil. Se entiende que las tecnologías han cambiado la forma en que los seres humanos interactúan y piensan en relación con el mundo que los rodea y la Educación Matemática necesita adaptarse a esta realidad. El problema que impulsa el estudio con las Tecnologías Digitales, del grupo GECEM, es: ¿Cuáles son las posibilidades didácticas de las tecnologías digitales para la educación matemática en la educación básica y superior? Se ha llevado el análisis sobre el potencial pedagógico de las tecnologías digitales (computadoras, tabletas, teléfonos inteligentes, calculadoras) en educación matemática, tanto en educación básica como en educación superior (formación de docentes y cursos que utilizan las matemáticas como apoyo en la instrucción profesional). La metodología utilizada durante la investigación fue de tipo exploratorio, de naturaleza analítica-descriptiva. Presentamos un simulador de un brazo robótico, un objeto de aprendizaje para la visualización del área de triángulos y una secuencia didáctica, con el tema Estadística y Educación Ambiental. Los resultados encontrados, con el uso de recursos digitales, son positivos y demuestran potencial para ser utilizados por los maestros en el aula, de forma tal que pueden explorarse en la planificación didáctica, tanto en educación básica como en la capacitación de maestros.

Palabras clave: Enseñanza de las matemáticas; enseñanza multimedia; estrategias educativas; STEM; Simuladores de Robótica 


\section{Introdução}

Este artigo apresenta os resultados de pesquisa do projeto Educação Matemática e Tecnologias Digitais, do Grupo de Estudos Curriculares de Educação Matemática (GECEM) vinculado ao Programa de Pós-Graduação em Ensino de Ciência e Matemática (Mestrado e Doutorado) da Universidade Luterana do Brasil (ULBRA), no município de Canoas, do estado do Rio Grande do Sul, Brasil.

$\mathrm{O}$ GECEM está registrado no $\mathrm{CNPq}-$ Conselho Nacional de Pesquisa, desde 2006, atuando em pesquisa na área de Currículo de Matemática nos aspectos: o que ensinar, como ensinar, quando ensinar e o que, quando, como avaliar, nos níveis fundamental, médio e superior, abrangendo a formação inicial e continuada de professores de Matemática. O objetivo geral que orienta o desenvolvimento das investigações do grupo GECEM é refletir sobre critérios e possibilidades que possam nortear uma transformação curricular em Matemática tendo como pressuposto básico o desenvolvimento de competências, nos estudantes da Educação Básica, que permitam uma participação cidadã, ativa e comprometida na sociedade em que se inserem, considerando teorias pedagógicas, didáticas e de ensino e aprendizagem da Matemática.

Neste sentido as pesquisas do grupo ligadas ao uso das Tecnologias Digitais de Informação e Comunicação (TDIC) são importantes e necessárias para dar sentido inovador, com foco no fazer do estudante, buscando desenvolver o pensamento matemático, a criatividade e um conhecimento científico consistente dos conceitos matemáticos, que podem auxiliá-lo à continuação dos estudos, à vida em sociedade, à vida profissional e pessoal. As investigações dos pesquisadores do GECEM sobre o potencial pedagógico das TDIC, utilizando Computadores, Tablets, Smartphones, Calculadoras, para a Educação Matemática, tanto na Educação Básica quanto no Ensino Superior (Formação de professores e cursos que utilizam a Matemática como suporte na formação profissional) buscam responder a questão: Quais as possibilidades didáticas das TDIC para a Educação Matemática na Educação Básica e Ensino Superior?

Neste artigo abordam-se alguns resultados das pesquisas realizadas pelo GECEM, apontando a importância das TDIC para um processo de ensinar Matemática de acordo com as exigências do mundo contemporâneo.

Importante salientar que serão utilizados, no decorrer do texto, os acrônimos TDIC para Tecnologias Digitais da Informação e Comunicação e TIC para Tecnologias da Informação e Comunicação, conforme utilizado pelos autores citados.

\section{$O$ professor de Matemática $\mathrm{e}$ as Tecnologias Digitais}

Em uma sociedade de bases tecnológicas, com mudanças contínuas, não é mais possível desprezar o potencial pedagógico que as TDIC apresentam quando incorporadas à educação. A integração dos recursos tecnológicos na Educação mostra-se irremediavelmente associada à necessidade de reforço da profissionalização docente e de uma (re) organização das dinâmicas escolares (Nóvoa, 2007). Segundo o autor torna-se importante perceber que ações se mostram necessárias para promover a efetiva inclusão das Tecnologias da Informação e Comunicação (TIC) no contexto escolar, mais especificamente, estudos de como se pode promover o desenvolvimento profissional docente para trabalhar, com eficiência e 
sustentabilidade esta inclusão no planejamento escolar.

Segundo Caccuri (2016) é importante que os professores sejam competentes para ensinar e aprender em um contexto de cultura digital. Entendendo cultura digital como um conjunto de saberes, crenças, usos e costumes que surgem na sociedade a partir da convergência das TIC, gerando uma revolução nos modos de acumular, apropriar-se e transmitir os dados, impulsionando novos desenvolvimentos sociais, políticos, econômicos e educativos.

Entende-se que os professores passam por diferentes etapas de integração das TDIC no planejamento didático, que abarcam desde o uso exploratório até o desenvolvimento de atitudes criativas e inovadoras para sua implementação como parte da tarefa educativa. Estas etapas, segundo Caccuri (2016), são:

1. Fase de exploração - a primeira abordagem, depois de superar os medos e de abrir a mente para as possibilidades que as TDIC oferecem para a Educação. Nesta etapa o professor introduz alguns recursos digitais no seu planejamento e se surpreende frente as opções possíveis. Já é capaz de desenvolver atividades de aprendizagem utilizando recursos digitais, ferramentas informáticas e meios audiovisuais. Já considera a internet como uma poderosa fonte de informação e utiliza ferramentas nas tarefas profissionais (planejamentos, controle das avaliações, entre outras).

2. Fase de integração - nesta fase o professor já utiliza as TDIC de forma autônoma, por meio do aprofundamento e da integração criativa de recursos informáticos. A partir de saberes e experiências prévias adquiridas pelo uso das
TDIC na vida cotidiana ou de seu uso na Educação na etapa de exploração, é consciente de potencial das TDIC e as integra na prática com segurança e confiança. Nesta fase é capaz de: planejar e publicar conteúdos digitais em diferentes formatos; acessar recursos disponíveis online para aprender de maneira não presencial e realiza cursos virtuais; participar de projetos colaborativos com outros colegas e com os seus alunos.

3. Fase de inovação - nesta etapa o professor está em condições de usar TDIC para criar, expressar ideias, construir conhecimentos de maneira colaborativa e desenvolver novas estratégias para reconfigurar a prática educativa. Sente-se capaz de panejar ambientes de aprendizagem virtuais, de adotar e adaptar novas ferramentas de uma múltipla variedade de fontes e recursos.

Segundo Pietrovzki (2015) é importante que os professores, ao planejarem suas aulas de Matemática com a integração das TDIC, sigam cinco recomendações:

1. Pensar o ensino da Matemática seguindo os enfoques próprios da didática da Matemática - ter clareza sobre qual a concepção de ensino e aprendizagem que se promove, qual é a ideia de sujeito (aluno) e o que significa para este enfoque saber matemática. Neste sentido, o professor propõe problemas para que os alunos produzam conhecimento partindo de seus saberes prévios e da busca pessoal de procedimentos que lhes permitam resolver as situações propostas;

2. Considerar as capacidades cognitivas cujo desenvolvimento se desejam promover nos estudantes - considerar as formas próprias da disciplina para a 
resolução das tarefas propostas, para interpretar, representar, definir, comunicar procedimentos e resultados tanto na forma oral como escrita e, requer o trabalho individual ou em colaboração com os seus pares (trabalho em grupos);

3. Selecionar adequadamente os conteúdos que se desejam ensinar com apoio das TDIC - é importante conhecer as formas em que as TDIC limitam ou facilitam as representações, explicações e demonstrações dos conceitos e procedimentos na área da Matemática;

4. Eleger e aplicar estratégias pedagógicas que permitam aproveitar ao máximo as tecnologias disponíveis - conhecer os diferentes tipos de atividades e recursos tecnológicos para desenvolvê-las. Fazer uma seleção variada para considerar o tratamento de novos conteúdos, a possibilidade de praticar, produzir, criar, trabalhar colaborativamente. Ter presente o produto que se pretende obter, os papéis que o aluno e o professor assumirão e os critérios de avaliação que melhor se ajustam ao ensino proposto;

5. Buscar e selecionar os recursos tecnológicos e definir seu modo de uso em função das necessidades pedagógicas identificar os momentos da classe que podem ser apoiados ou potencializados com o recurso das TDIC. Uma vez estabelecidos para que se quer usar estes recursos, deve-se buscá-los, selecioná-los e definir as maneiras adequadas de utilizá-los.

As TIC e outras ferramentas digitais influenciam o currículo escolar, mudando a maneira de ensinar e influenciando no que se deve ensinar (NCTM, 2014). Porém, o uso eficaz das TIC requer conhecimento e um planejamento cuidadoso, exigindo dos docentes um desenvolvimento profissional adequado para poder utilizá-las em todo o seu potencial. Importante frisar que se o professor não conhece ou não está convencido da importância do uso destes recursos no desenvolvimento do processo de ensino e aprendizagem pode sentir-se inseguro a respeito do seu uso, não os incorporando ao currículo de um modo significativo.

Ressalta-se que se corrobora com as ideias de Bittar (2010) quanto a distinguir os termos inserção e integração das TDIC na Educação. Entende-se que professor insere as TDIC quando ela não provoca diferenças na aprendizagem, ou seja, a tecnologia é como se fosse um elemento estranho do fazer pedagógico. Entretanto, "integrar um novo instrumento (tecnologia digital) em sala de aula, implica mudanças pedagógicas, mudanças do ponto de vista da visão de ensino, que deve ser estudada e considerada pelos professores" (Bittar, 2010, p. 220).

Almeida e Valente (2011) salientam que a integração requer a reconstrução de conhecimentos por parte do estudante e, para tanto é necessário que o professor vivencie o processo de apropriação pedagógica das TDIC. Sob esse enfoque, o uso dos recursos das TDIC no processo de ensino e aprendizagem não ocorre apenas inserindo-os na sala de aula, mas integrando-os ao currículo, de modo a propiciar uma nova forma de ensinar (Bittar, 2010). A seguir discorre-se sobre o papel das TDIC na Educação Matemática.

\section{O papel das Tecnologias Digi- tais da Informação e Comuni- cação na Educação Matemática}

Para a OCDE (2010) o mundo é rico em tecnologia, fazendo surgir novos interesses para a educação e se espera que as 
escolas se tornem a vanguarda na sociedade do conhecimento. Entende que a tecnologia pode fornecer os instrumentos necessários para a melhoria do processo de ensino e aprendizagem, abrindo novas oportunidade e caminhos. Primeiro, aumentando a customização do processo de aprendizagem, adaptando-o às necessidades particulares do aluno. Segundo a educação tem o papel de preparar os estudantes para a vida adulta, e, consequentemente, deve fornecer aos estudantes as habilidades necessárias para se unirem a uma sociedade onde as competências ligadas à tecnologia tornam-se cada vez mais indispensáveis. A OCDE (2010) também afirma que em uma economia de conhecimento dirigido pela tecnologia, pessoas que não adquirem e não se apropriam destas competências podem sofrer de uma nova forma de separação digital, dificultando que se integrarem plenamente à economia e à sociedade do conhecimento.

Kampff et al (2004) afirmam que em uma sociedade de bases tecnológicas, com mudanças contínuas, não é mais possível desprezar o potencial pedagógico que as TIC apresentam quanto incorporadas a educação. Assim, os computadores, os Tablets, as calculadoras são instrumentos pertinentes no processo de ensino e aprendizagem, cabendo à escola utilizá-los de forma coerente, com uma proposta pedagógica atual e comprometida com uma aprendizagem significativa, que favoreça diferentes formas de construir o conhecimento.

As tecnologias têm alterado o modo de interação e de pensamento do ser humano em relação ao mundo que o rodeia. Neste período de informatização massiva, no qual as atividades têm migrado para o formato digital, a Educação, e a Educação Matemática, também necessitam adequar-se a essa realidade. Com os avanços tecnológicos, a redução dos custos envolvidos tem facilitado o acesso à tecnologia; contudo, além do acesso, é preciso conhecimento para utilizá-la em todo o seu potencial (Homa e Groenwald, 2016).

A evolução tecnológica, segundo Kenski (2012), não se restringe apenas aos usos de equipamentos e produtos. Para a autora o uso de determinada tecnologia impõe-se à cultura existente e transforma o comportamento individual e social, transformando suas maneiras de pensar, sentir, agir, as maneiras de se comunicar e de adquirir conhecimentos, criando uma cultura e um novo modelo de sociedade. Kenski (2012) afirma que as pessoas estão vivendo em um novo momento tecnológico, baseado na cultura digital.

Inserir-se na sociedade da informação não quer dizer apenas ter acesso à TIC, mas principalmente, saber utilizar essa tecnologia para a busca e a seleção de informações que permitam a cada pessoa resolver os problemas do cotidiano, compreender o mundo e atuar na transformação de seu contexto (Almeida, 2008). Neste sentido, para o NCTM (2014) todos os educandos deveriam ter acesso às tecnologias e a outras ferramentas que respaldem o ensino e a aprendizagem da Matemática.

Segundo Borba et al (2016, p. 9), "as dimensões da inovação tecnológica permitem a exploração e o surgimento de cenários alternativos para a educação e, em especial, para o ensino e aprendizagem da Matemática", cabendo ao professor explorar e proporcionar situações para a aprendizagem com o uso das tecnologias.

Desenvolver o processo de ensino e aprendizagem da Matemática no século XXI é um desafio, para os professores, para os alunos e, também, para as famílias que devem acompanhar este processo (Pietrovzki, 2015). Para a autora as questões inerentes ao 
ensino da Matemática são: Que Matemática ensinar, quais metodologias utilizar, que ferramentas são as mais apropriadas, como envolver os alunos no processo de estudo para alcançar uma aprendizagem significativa?

Entende-se que a integração das TDIC pode potencializar o processo de ensino e aprendizagem, pois favorece a criação de ambientes de aprendizagem dinâmicos, centrados no estudante com uma variada gama de atividades criando situações que possibilitam o desenvolvimento das diferentes habilidades requeridas para uma aprendizagem de qualidade. Logo o planejamento de um tema utilizando TDIC deve considerar tanto os aportes da didática da Matemática (resolução de problemas, modelagem matemática, simulações matemáticas, jogos, projetos interdisciplinares), como os aportes dos modelos e marcos gerais para o ensino com integração das TDIC.

Segundo o NCTM (2014) para uma aprendizagem significativa da Matemática, as ferramentas e as tecnologias devem ser consideradas como características indispensáveis para a sala de aula, considerando que estes recursos podem ser utilizados para reunir dados, fazer pesquisas na sala de aula e para utilizar aplicações que façam cálculos, simulações, assim como para fomentar a visualização, permitindo que os alunos se envolvam com atividades que exijam habilidades para resolução de problemas. Os Computadores, tablets, smartphones e calculadoras tornam acessíveis uma gama de aplicativos que auxiliam aos usuários explorarem Matemática, dando sentido aos conceitos e procedimentos, envolvendo-os com o raciocínio matemático (NCTM, 2014). Também, afirma que as TIC são uma poderosa ferramenta para fazer Matemática, que o emprego das TIC pode ajudar aos estudantes a visualizar e compreender importantes conceitos matemáticos, respaldando seu raciocínio matemático e sua capacidade para resolver problemas.

Segundo a Lei de Diretrizes e Bases da Educação Nacional (Ministério da Educação, 1996), a Educação Nacional tem por finalidade o pleno desenvolvimento do educando, seu preparo para o exercício da cidadania e sua qualificação para o trabalho. Deste modo, a Educação e a inserção na sociedade digital implicam em uma adequação da sala de aula à realidade tecnológica, cujo uso da tecnologia pelos docentes é condição necessária para tal.

Embora o Ministério da Educação (Ministério da Educação, 2013) considere importante a utilização de tecnologias de qualidade objetivando a melhoria da Educação, o mesmo adverte que o uso de recurso tecnológico, de forma isolada e desalinhada com a proposta pedagógica da escola, não garante a qualidade da Educação. Ao utilizar as tecnologias para proporcionar condições favoráveis à aprendizagem, o professor deve, antes de tudo, definir o objetivo instrucional desejado para então organizar as ações e recursos para atingi-lo, sendo fundamental conhecer as possibilidades que as tecnologias oferecem e quais tecnologias são adequadas aos estudantes, ao conteúdo a ser desenvolvido e ao nível de ensino a que se destina.

De acordo Ramos et al (2011), a consequência mais importante do uso de tecnologias digitais no ensino "será talvez a emergência de novos territórios nos domínios dos conteúdos e recursos educativos digitais, novos conceitos, novas ferramentas de produção e de novos produtores" (Ramos et al, 2011, p. 15).

Perrenoud (2000), com base no pensamento de Tardif, salienta que as TIC demandam e, ao mesmo tempo, oportunizam uma mudança de paradigma, em relação às 
aprendizagens e não às tecnologias. Para o autor as TIC contribuem com os trabalhos pedagógicos e didáticos porque permitem criar situações de aprendizagem diversificadas.

As atividades planejadas com TIC devem possibilitar que os estudantes investiguem os problemas e as ideias matemáticas, que de outra forma seriam muito difíceis de explorar ou que tomariam muito tempo (NCTM, 2014). Para a aprendizagem é fundamental a participação, o trabalho colaborativo, a interatividade entre os estudantes, com a discussão e a troca de ideias, o acesso à informação e a pesquisa em um ambiente propício para que todas essas ações aconteçam de forma integrada e simultânea.

Para Valente (2014), a ação educacional deve possibilitar a construção do conhecimento pelo aprendiz. Desse modo, é fundamental que sejam criados ambientes de aprendizagem em que ocorram tanto aspectos de transmissão como de construção do conhecimento que impliquem na atribuição de significado, ou seja, apropriação da informação.

Assim, a questão fundamental no processo educacional "é saber como prover a informação de modo que ela possa ser interpretada pelo aprendiz que passa a entender quais ações ele deve realizar para que a informação seja convertida em conhecimento" (Valente, 2014, p. 144). Para o referido autor, um caminho possível para o alcance desse objetivo é a utilização das TDIC, pois estas podem tornarem-se ferramentas cognitivas significativas.

Uma das diretrizes da Base Nacional Comum Curricular - BNCC (Ministério da Educação, 2018) é a inserção das tecnologias, aliada ao protagonismo do estudante na sociedade em que vive, enfatizando a utilização de TDIC e o desenvolvimento de competências e habilidades relacionadas ao uso crítico e responsável das mesmas, incentiva, também, a modernização dos recursos e das práticas pedagógicas com o objetivo de formar as habilidades e competências necessárias ao século XXI.

Com este enfoque teórico as investigações no GECEM foram desenvolvidas, aplicadas e avaliadas.

\section{Percurso Metodológico}

A metodologia utilizada nas pesquisas do GECEM foi do tipo exploratória de natureza analítico-descritiva, em que o pesquisador busca maiores informações sobre determinado assunto investigado, bem como a familiarização para obter novas percepções do fenômeno estudado (Thomas \& Nelson, 2002).

Os envolvidos no processo de investigação foram: os pesquisadores do GECEM (professores do PPGECIM, alunos de mestrado e doutorado, alunos de iniciação científica do curso de Matemática Licenciatura da ULBRA); professores de Matemática, do município de Canoas, participantes de um grupo de formação continuada (10 professores de Matemática que se reuniram mensalmente na ULBRA).

Para o desenvolvimento dos objetos de aprendizagem foram realizadas reuniões semanais com o grupo de investigação GECEM, para discussão e reflexão dos recursos pesquisados e/ou desenvolvidos; reuniões mensais com o grupo de formação continuada, para a análise das atividades planejadas e estudos das possibilidades de uso das mesmas; aplicação das atividades e análise dos resultados através das observações realizadas no grupo de formação continuada de professores de Matemática; replanejamento das atividades propostas ao grupo de professores incorporando suas sugestões e reflexões sobre o uso das mesmas. 
A seguir apresentam-se exemplos de objetos de aprendizagem ou recurso educacional digital (RED) desenvolvidos nas pesquisas realizadas pelos componentes do GECEM.

\section{Exemplos de situações didáti- cas com Tecnologias Digitais}

Um recurso educacional digital (RED), cujos elementos possibilitam a modelação, a simulação, a animação, a combinação multimídia, a interatividade (que pode assumir formas diferentes), favorece a elaboração de estratégias de ensino e modos de aprendizagem diversificadas e que podem ser orientadas à aprendizagem de conceitos e teorias por meio de uma mescla de imagens, palavras e sons, entre outros (Ramos et al, 2011).

Deste modo um recurso educacional digital deve possuir um conceito ou conteúdo completo em si. Para Willey (2002), objetos de aprendizagem são recursos digitais que podem ser reutilizados para o suporte ao desenvolvimento do processo de ensino e aprendizagem; para o IEEE (Institute of Electrical and Electronics Engineers, 2002) os objetos de aprendizagem são qualquer entidade, digital ou não, que pode ser utilizada, reutilizada ou referenciada durante o processo de ensino e aprendizagem que utilize tecnologia.

Dentro do contexto educacional, é importante a característica de reutilização para um melhor aproveitamento dos objetos de aprendizagem desenvolvidos. Sendo imprescindível uma descrição mínima de seus objetivos, tipos de interações, e que este exista em si só, ou seja, não depender de outros objetos para atender ao objetivo proposto.

Na elaboração de atividades que utilizam de objetos de aprendizagem as mesmas devem ser compostas de instruções para que os alunos entendam quais são as interações disponíveis e o que cada informação representa, de modo que cada objetivo seja alcançado dentro de aproximadamente 10 minutos pois, segundo Mortimer (2002), um objeto de aprendizagem deve ter definido o tempo de execução para que o objetivo de aprendizagem alcançado.

Merrill (2002) afirma que objetos sem um design instrucional são somente objetos de conhecimento, ou seja, tem um caráter mais informativo, por isso as atividades organizadas como sequências didáticas necessitam seguir uma abordagem construtivista, na qual o estudante através das interações que realiza com os objetos de aprendizagem identifica as relações e generaliza os conceitos propostos.

Entende-se por sequência didática a organização de um conteúdo qualquer, a partir da articulação entre os conceitos e procedimentos a serem desenvolvidos, com atividades didáticas planejadas para esse fim, com foco na aprendizagem. Segundo Zabala (1998, p. 18), sequências didáticas são "[...] um conjunto de atividades ordenadas, estruturadas e articuladas para a realização de certos objetivos educacionais, que tem um princípio e um fim conhecido, tanto pelos professores como pelos alunos". Dolz e Schneuwly (2004) consideram que sequências didáticas são organizadas pelo professor com o objetivo de alcançar a aprendizagem de seus alunos, e envolvem atividades de aprendizagem e avaliação.

O uso de sequências didáticas disponibilizadas em plataformas de ensino, segundo Groenwald et al. (2009, p.2) possibilitam a utilização de diferentes recursos, com padrão superior de qualidade, como vídeos-exemplo, textos e animações, ou seja, um conteúdo com maior qualidade visual. 


\section{Exemplo de um Simulador de braço robótico}

Apresenta-se um objeto de aprendizagem na forma de um simulador de um braço robótico simplificado de cinco para três graus de liberdade. O objetivo do simulador é que o aluno compreenda e realize as conversões das representações retangular para polar, podendo este objeto de aprendizagem ser utilizado em sequencias didáticas para a aprendizagem da trigonometria ou da representação dos números complexos.

Para a movimentação do braço robótico o aluno deve identificar o ângulo de rotação e o ângulo da articulação central do braço robótico que se converte na distância entre a base do braço robótico e a bola que o braço deve pegar (Homa, 2019). Esse movimento requer que o aluno utilize a representação polar, para que o braço robótico realize a ação de pegar a bola vermelha, mas, além do ângulo de rotação e a distância o simulador requer o uso de outros conhecimentos de trigonometria. Para o cálculo do ângulo da articulação do braço, o aluno utiliza o teorema de Pitágoras associado a soma dos ângulos internos do triângulo, isto por que os dois segmentos têm o mesmo comprimento, logo forma-se um triângulo isósceles entre os segmentos do braço robótico e o segmento que representa a distância da base à bola (Figura 1).

Caso o aluno esteja familiarizado com a Lei dos Cossenos é possível aplicá-la diretamente para o cálculo do ângulo de abertura que tem a distância entre a base e a bola como o lado oposto ao ângulo.

O simulador foi desenvolvido no GeoGebra, que além do recurso de visualização dos objetos geométricos envolvidos, é um CAS (Computer Algebra System) podendo ser utilizado para os cálculos necessários. Apresentam-se os cálculos referidos na Figura 2.

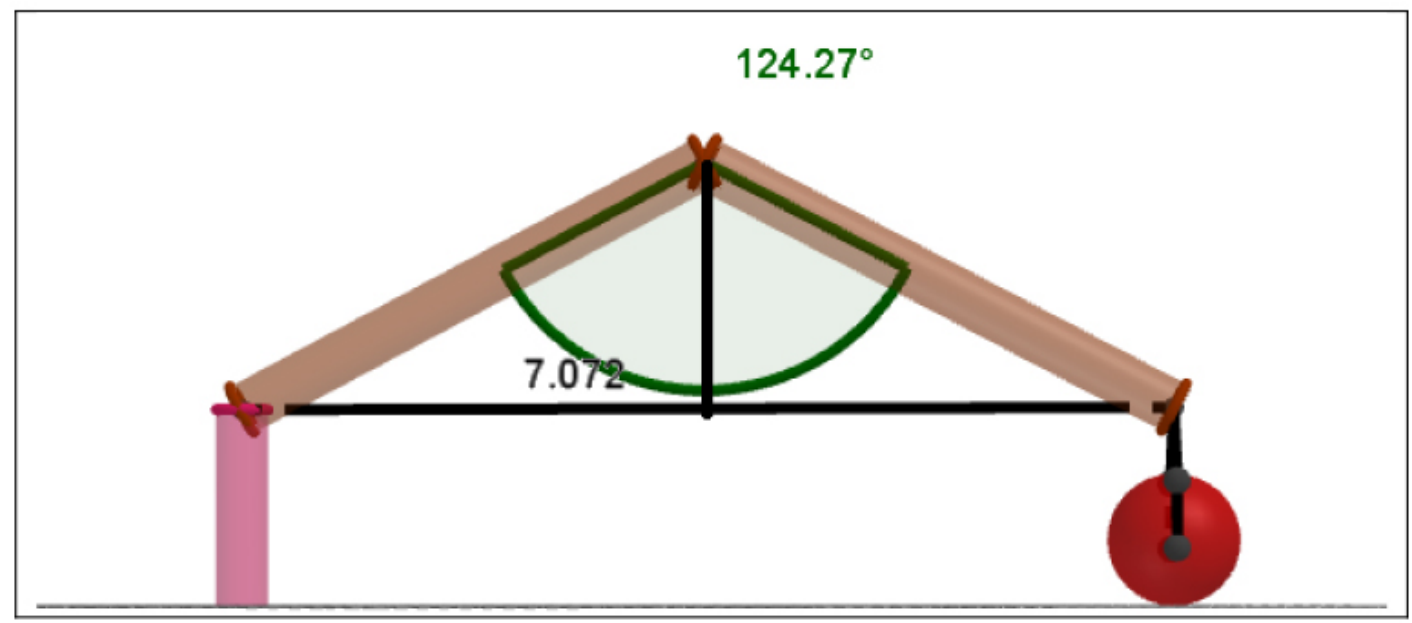

Figura 1 - Posição do braço robótico ao pegar a bola, Homa (2019). Extraído de Robotics Simulators in STEM Education. Acta Scientiae. 21(5), 178-191. 


\begin{tabular}{|c|c|c|}
\hline \multicolumn{2}{|c|}{ Cálculo Simbólico (CAS) } & \multirow{6}{*}{$\begin{array}{l}\text { Comandos do CAS } \\
\text { O comando calcula } \sqrt{5^{2}+5^{2}} \\
\text { Cálculo do ângulo oposto ao segmento do triân- } \\
\text { gulo que representa a distância da base à bola. } \\
\text { Cálculo para a conversão de radianos em graus } \\
\text { O ângulo de rotação é dado por } \\
\operatorname{arcsen~}\left(\frac{3}{5830951894845}\right)\end{array}$} \\
\hline 1 & $\operatorname{sqrt}\left(5^{\wedge} 2+5^{\wedge} 2\right)$ & \\
\hline 0 & $\approx 7.071$ & \\
\hline 2 & $7.071^{\wedge} 2=4^{\wedge} 2+4^{\wedge} 2-2^{*} 4^{\star} 4^{*} \cos (x), x=1$ & \\
\hline 0 & ResolverNumericamente: $\{x=\mathbf{2 . 1 6 8}\}$ & \\
\hline 3 & $2.168^{\star} 180 / \mathrm{pi}$ & \\
\hline 0 & $\approx 124.217$ & \\
\hline 4 & $\operatorname{sen}^{-1}(5 / 7.071)$ & \\
\hline 0 & $\approx 0.785$ & Cálculo para a conversão de radianos em graus \\
\hline 5 & $0.7854077535814^{*} 180 / \mathrm{pi}$ & \\
\hline 0 & $\approx 45.001$ & \\
\hline
\end{tabular}

Figura 2 - Comandos do CAS para cálculo do ângulo de abertura e rotação do braço robótico, Homa (2019). Extraído de Robotics Simulators in STEM Education. Acta Scientiae. 21(5), 178-191.

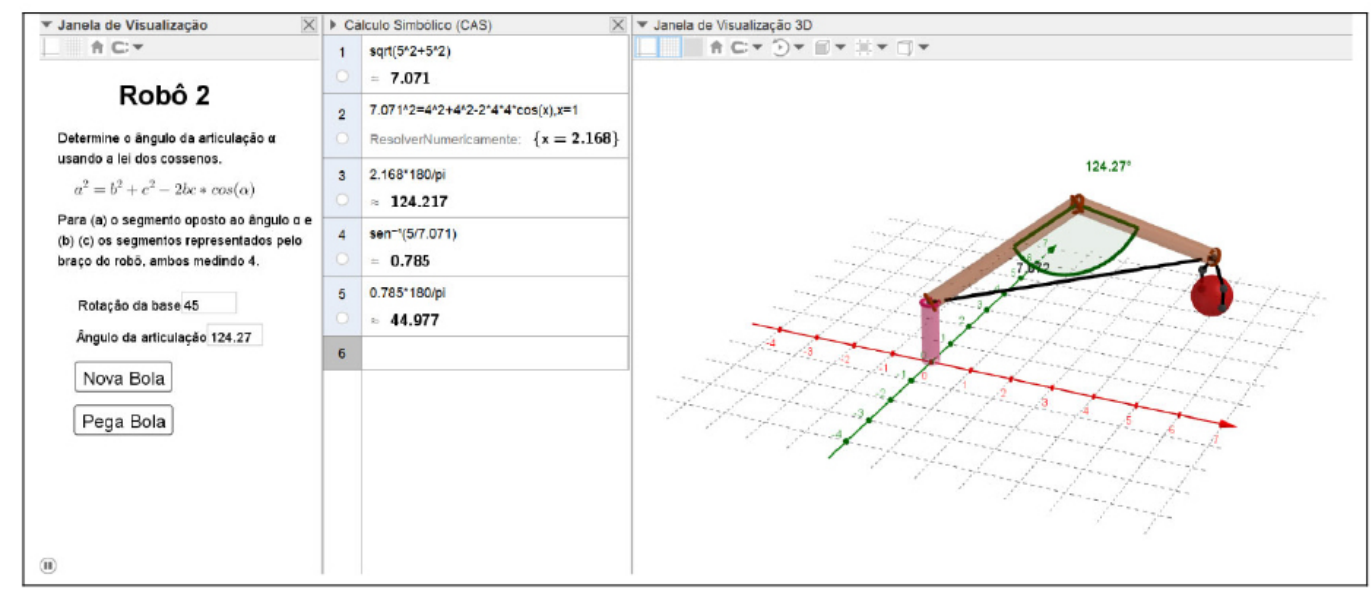

Figura 3 - Simulador robótico 2 para aprendizagem da representação polar, Homa (2019). Extraído de Robotics Simulators in STEM Education. Acta Scientiae. 21(5), 178-191.

Na Figura 3 tem-se uma situação com a bola na coordenada $(5,5)$ que por sua vez está a uma distância de 7,071 da base na origem, que pela Lei dos cossenos:

$$
7,071^{2}=4^{2}+4^{2}-2 * 4 * 4 * 4 \cos (x)
$$

Utilizando o CAS para o cálculo do ângulo tem-se o valor de $x$ em 2.168 radianos, sendo necessária a conversão para ser informado ao simulador o valor do ângulo em graus. Isto leva os estudantes a buscarem o conhecimento necessário para a conversão através do uso da razão e proporção:

$$
\begin{gathered}
\frac{\text { ângulo em graus }}{180^{\circ}}=\frac{2.168}{\pi} \\
\text { ângulo em graus }=\frac{2.168 * 180^{\circ}}{\pi}
\end{gathered}
$$


Para simplificar os comandos necessários para a movimentação do braço, o movimento transversal do segmento ligado a base e da garra para que a mesma permaneça perpendicular ao plano são gerenciados pelo próprio simulador. Ao diminuir o grau de realismo em um simulador, busca-se deixá-lo adequado aos conhecimentos do aluno que trabalha com o mesmo.

\section{Objeto de aprendizagem - Área de triângulos}

Outro objeto de aprendizagem, desenvolvido pelos pesquisadores do GECEM, construído no software GeoGebra, para a visualização da área de triângulos, é apresentado na Figura 4. Neste objeto é possível que o estudante visualize a transformação do triângulo em um paralelogramo e identifique que a medida da área do triângulo é a metade da área do paralelogramo. Ressalta-se que a sequência didática trabalha a área de quadrados, retângulos e paralelogramos, antes da área do triângulo. Este objeto permite que o estudante realize as transformações optando por um dos lados que será considerado como a base do triângulo, deste modo o estudante observa que, dependendo da base escolhida, obtém-se diferentes alturas, mas a área permanece inalterada.

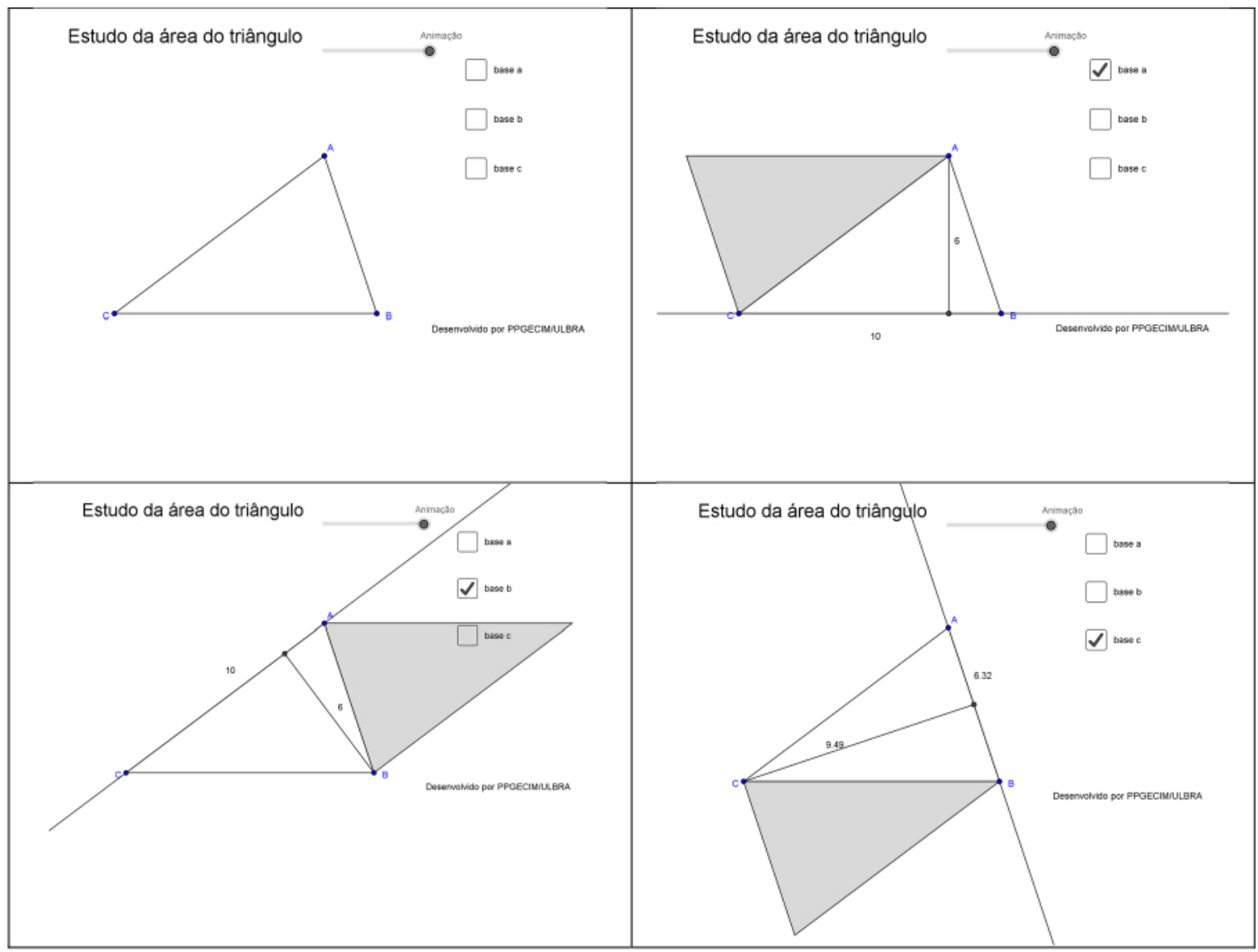

Figura 4 - Objeto de Aprendizagem Area de Triângulo. Nota: Fonte os autores. 


\section{Sequências didáticas}

Apresenta-se o exemplo de uma sequência didática com o tema Estatística e Educação Ambiental. O mesmo está disponibilizado na plataforma de ensino, denominada Sistema Integrado de Ensino e Aprendizagem (SIENA), no endereço http://siena.ulbra.br. Em cada sequência desenvolvida no SIENA, são necessárias as seguintes ações: construção do grafo com os conceitos desenvolvidos na sequência didática; banco de itens para os testes adaptativos associados aos conceitos; objetos de aprendizagem apresentados para a aprendizagem dos conceitos definidos.

O grafo do projeto Estatística e Educação Ambiental é composto por 5 conceitos: Introdução à Estatística, Tabelas, Gráficos, Medidas de Tendência Central e Resolução de problemas, conforme a figura 5 .
A organização do grafo é do tipo botton-top, ou seja, o planejamento é desenvolvido, de baixo para cima, indo dos objetivos primários, para os secundários até atingir o objetivo de aprendizagem definido para a sequência didática.

O teste adaptativo da plataforma possui certo grau de inteligência, pois apresenta questões com dificuldades adequadas ao conhecimento inferido sobre o avaliado. Deste modo o banco de itens de avaliação (questões) deve ter um número maior de itens que o apresentado ao avaliado. Considera-se adequado 30 questões para cada conceito do grafo, classificadas em 10 fáceis, 10 médias e 10 difíceis. Na figura 6, apresentam-se três questões dos testes, uma questão de cada nível de dificuldade, do conceito de Introdução a Estatística. Em média, nos experimentos realizados, o conhecimento do estudante é inferido com 10 itens.

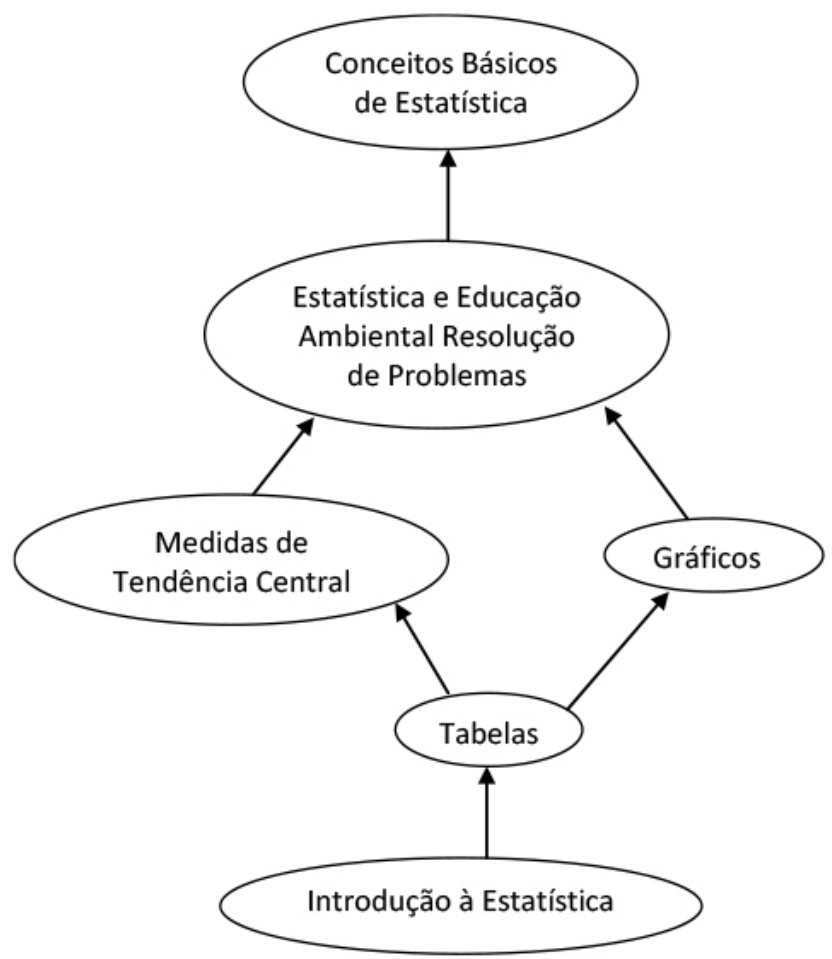

Figura 5-Grafo com os conceitos de Estatística. Nota: Fonte http://siena.ulbra.br. 


\begin{tabular}{|c|c|c|}
\hline nível fácil & Questão de nível médio & Qu \\
\hline $\begin{array}{l}\text { Assinale a alternativa que contém } \\
\text { os objetivos da Estatística: } \\
\text { a) Resumir dados para realizar } \\
\text { pesquisas. } \\
\text { b) Apenas interpretar dados. } \\
\text { c) Obter, organizar e analisar da- } \\
\text { dos, determinar as correlações } \\
\text { que apresentam. } \\
\text { d) Apenas coletar dados. } \\
\text { e) Criar tabelas e gráficos. }\end{array}$ & $\begin{array}{l}\text { Ficha de Estudo } \\
\text { Nomedo animal: } \\
\text { Sexo: } \\
\text { Idade: } \\
\text { Atura: } \\
\text { Peso: } \\
\text { Més da obsernacio: } \\
\text { Pesquisadores do Instituto Amigos } \\
\text { do Urso têm estudado o desenvolvi- } \\
\text { mento de ursos marrons selvagens } \\
\text { que vivem em uma certa floresta do } \\
\text { Canadá. O objetivo do projeto é estu- } \\
\text { dar algumas características dos ursos. } \\
\text { A ficha de coleta de dados representa- } \\
\text { da na figura mostra as características } \\
\text { estudadas. De acordo com os dados } \\
\text { da ficha de estudos, podemos classifi- } \\
\text { car como variáveis qualitativas: } \\
\text { a) Idade e altura } \\
\text { b) Sexo e peso } \\
\text { c) Altura e peso } \\
\text { d) Sexo e mês de observação } \\
\text { e) Mês da observação e peso }\end{array}$ & $\begin{array}{l}\text { A Usina Termoelétrica de Candiota } \\
\text { II despeja na atmosfera, diariamen- } \\
\text { te, cerca de } 45 \text { toneladas de enxo- } \\
\text { fre. Cada } 100 \text { toneladas de carvão } \\
\text { que são queimadas para a geração } \\
\text { de energia elétrica, produzem uma } \\
\text { tonelada de enxofre. Assinale a al- } \\
\text { ternativa que contém o tipo de Es- } \\
\text { tatística utilizado nesta informação: } \\
\text { a) Estatística da População } \\
\text { b) Estatística da Amostra } \\
\text { c) Estatística Descritiva } \\
\text { d) Estatística Inferencial } \\
\text { e) Estatística Grupal }\end{array}$ \\
\hline
\end{tabular}

Figura 6 - Exemplos de questões do teste Introdução aos conceitos de Estatística. Nota: Fonte http://siena.ulbra.br.

Para as sequências didáticas foram utilizados os seguintes recursos informáticos: o PowerPoint, editor de apresentações gráficas da Microsoft; o Ispring, para converter as apresentações ppt em vídeos html5 e flash; o JClic, um programa para a criação, realização e avaliação de atividades educativas multimídia, para o desenvolvimento das atividades lúdicas; jogos online; sites informativos.

A sequência das atividades não é fechada, isto é, permite que o estudante selecione a atividade que deseja realizar. Para isso há um menu de seleção com os links de cada atividade, que permite ao aluno estudar conforme sua preferência, ou seguir a ordem sugerida, conforme se apresenta na figura 7 o menu de atividades

\section{Estatística e Educação Ambiental \\ Gráficos \\ Clique no círculo 1 para iniciar o estudo. Depois siga ordem até terminar.}
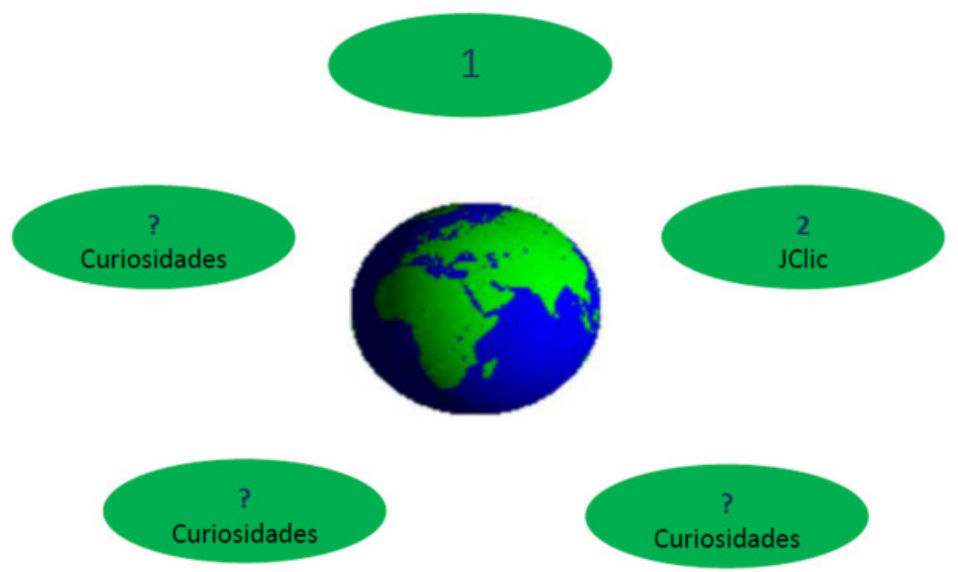

Figura 7 - Menu do conceito de Gráficos.

Nota: Fonte http://siena.ulbra.br. 
para a aprendizagem de Gráficos do projeto Estatística e Educação Ambiental. Os itens numerados indicam a ordem das atividades que a serem realizadas, os demais são livres para escolha do aluno.

As histórias em quadrinhos são recursos que permitem uma contextualização dos problemas utilizando textos e imagens que remetem a uma identificação da situação problema. O texto na forma de diálogos permite ressaltar o conteúdo como em uma conversa real, através de perguntas e animações para realçar as informações. A figura 8 é um exemplo de uma história em quadrinhos do conceito de Tabelas.

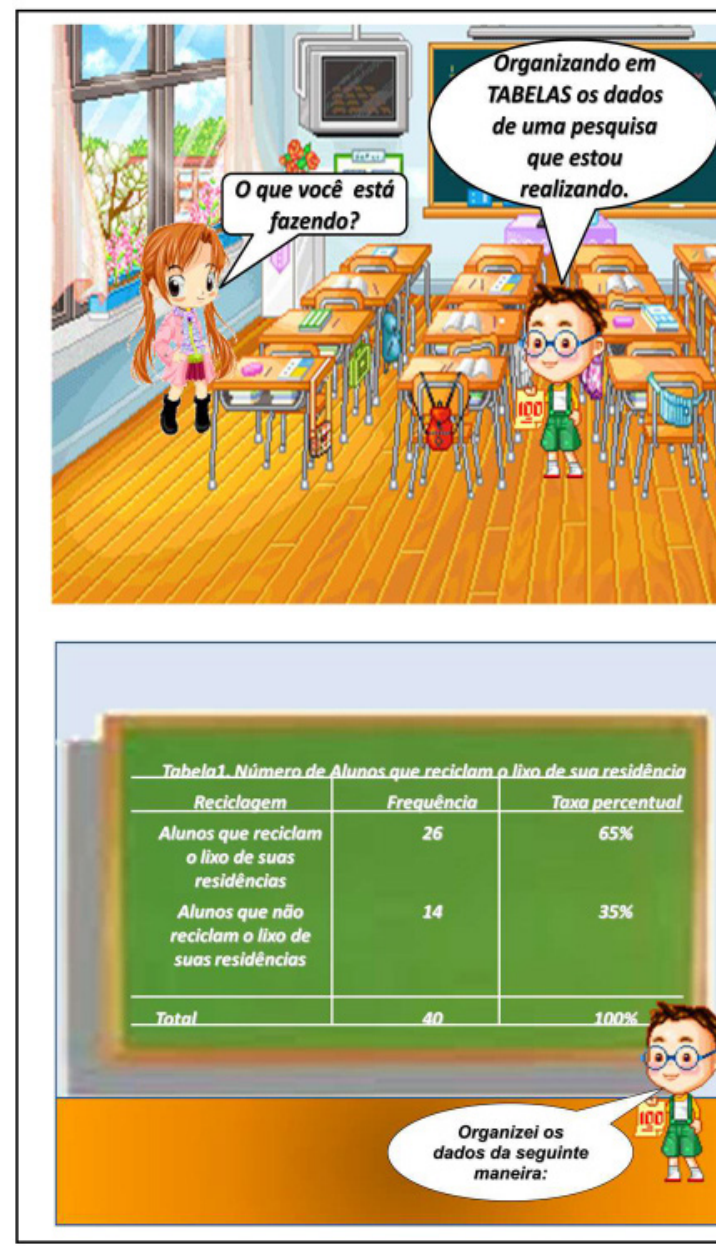

Figura 8 - Apresentação em HTML do con
O JClic, é um programa desenvolvido em Java que permite desenvolver atividades interativas e exportá-las para que sejam disponibilizadas na WEB. Um exemplo de atividade, desenvolvida no JClic, apresenta-se na Figura 9. A atividade é de associação na qual o estudante seleciona a imagem e a designação correta, diferente de uma questão avaliativa, a atividade pode ser realizada quantas vezes forem necessárias indicando que ocorreu um equívoco fazendo com que o aluno reveja seus conceitos sobre o tema. A atividade informa a quantidade de tentativas, o tempo e o número de itens associados equivocadamente. Tais informações podem ser utilizadas para indicar o nível de domínio do aluno sobre o assunto estudado.
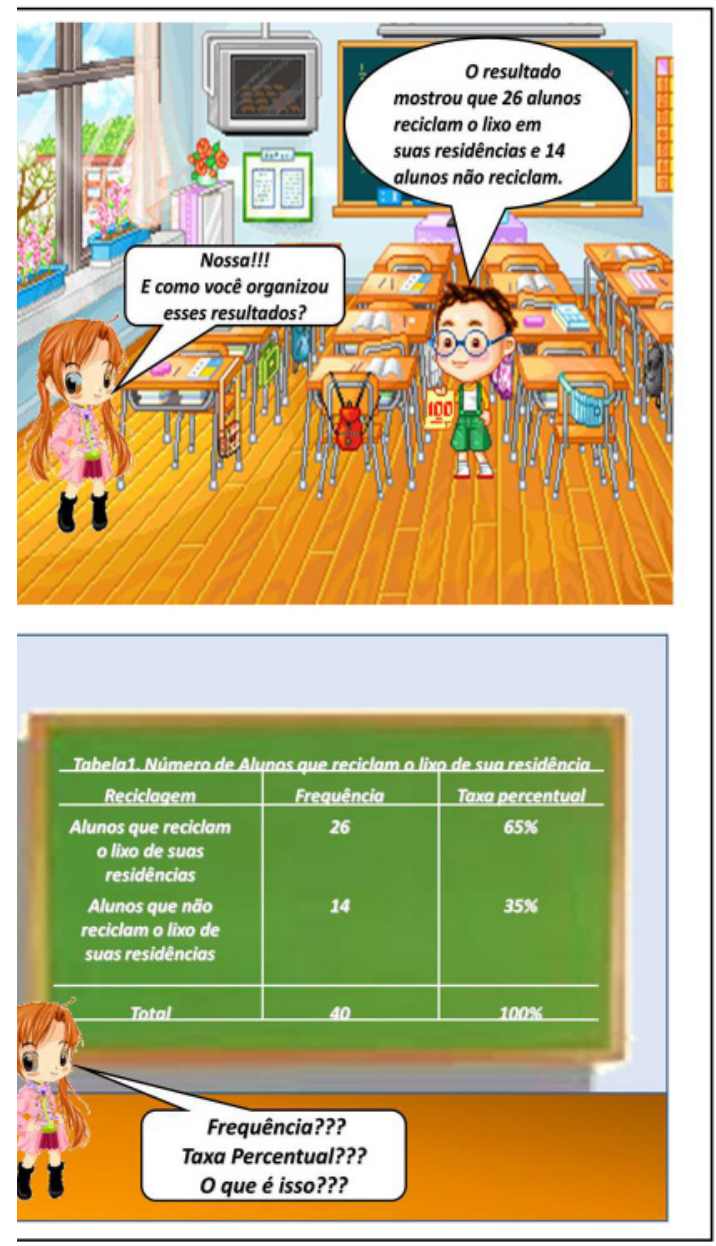

Tabelas. Nota: Fonte http://siena.ulbra.br. 


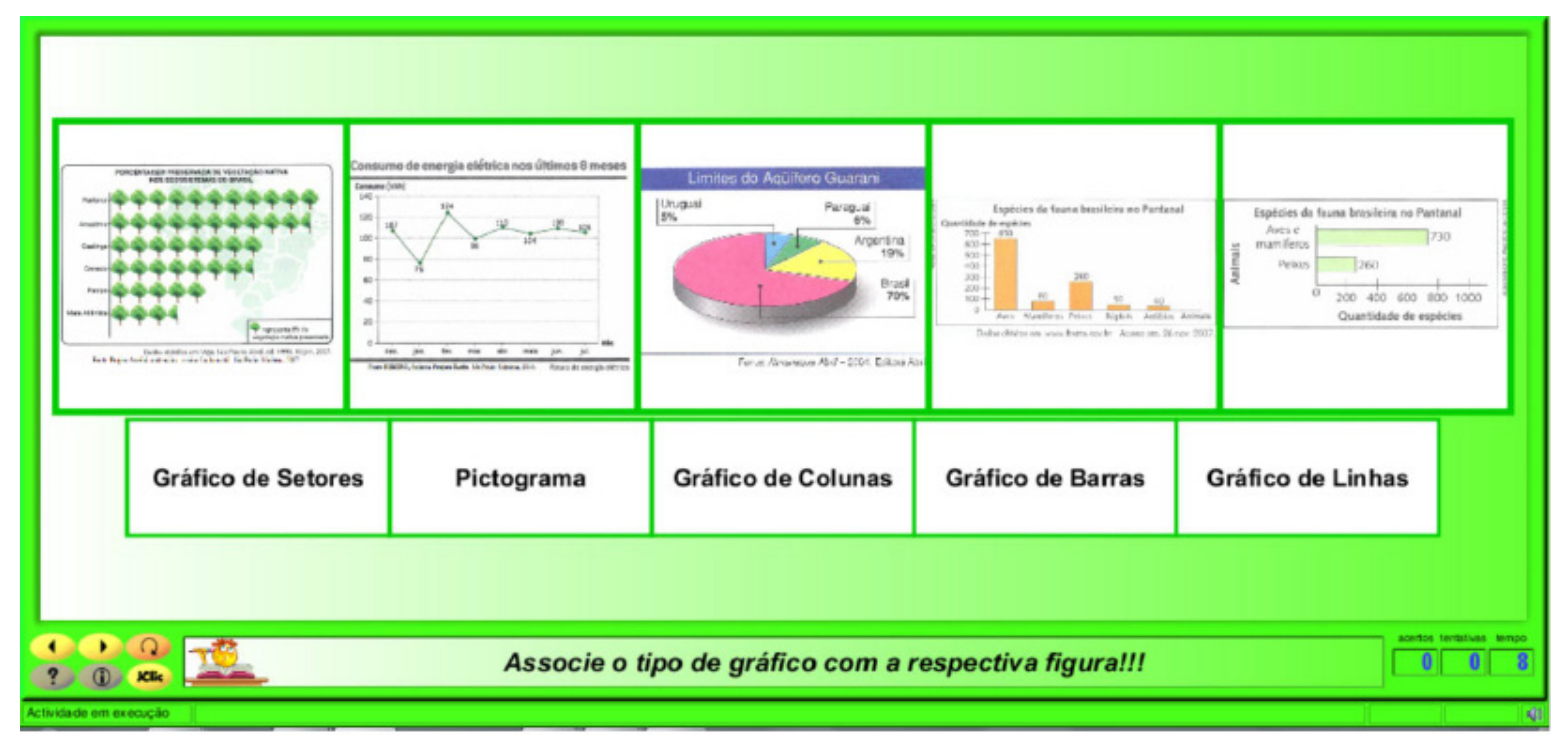

Figura 9 - Exemplo de atividade de Gráficos no JClic. Nota: Fonte http://siena.ulbra.br.

O projeto Estatística e Educação Ambiental foi implementado (desenvolvido, aplicado e avaliado) no mestrado defendido por Melo (2013), cujos resultados estão disponíveis em http://ppgecim.ulbra.br/teses.

\section{Considerações finais}

Para finalizar, ressalta-se que o professor deve estar preparado para inserir esses recursos em sala de aula, mas também não deve ter como objetivo utilizar a tecnologia apenas pelo uso, sem uma intenção clara e bem estruturada. Nesse sentido Barboza Jr (2009) ressalta que as tecnologias fornecem vários recursos que podem ser aplicados na educação, porém cada um desses recursos deve ser estudado e analisado pelos professores antes de serem usados em sala de aula, caso contrário, só servirá para informatizar o que era feito no modelo tradicional de educação.

Os resultados encontrados, com a utilização dos Recursos Digitais, têm apresentado resultados positivos e demonstram um potencial a serem utilizados pelos professores em sala de aula, podendo ser explorados no planejamento didático, tanto na Educação Básica quanto em cursos de formação de professores.

Salienta-se, também, que os softwares como o Geogebra e o JClic são adequados ao desenvolvimento de atividades interativas sem que seja necessário o conhecimento de programação avançada, sendo assim ferramentas

Importante frisar que os objetos desenvolvidos não devem ser apresentados individualmente, pois os mesmos são baseados no conhecimento matemático dos conceitos envolvidos, logo, salienta-se a importância da construção de uma sequência didática que apresente os objetos encadeados, de forma que permitam a visualização, o desenvolvimento de conjecturas e a generalização por parte dos estudantes. 


\section{Referências}

Almeida, M. E. B. (2008). Tecnologia na escola: criação de redes de conhecimentos. Tecnologias na Escola, 71-73. http://portal.mec.gov. br/seed/arquivos/pdf/2sf.pdf

Almeida, M.E.B.; Valente, J.A. (2011). Tecnologias e currículo: trajetórias convergentes ou divergentes? Dialogía, São Paulo, (17), 185-187. Recuperado de https://periodicos.uninove.br/index.php?journal $=$ dialogia\&page $=$ article $\&$ op $=$ view $\&$ path $\%$ $5 \mathrm{~B} \% 5 \mathrm{D}=4199 \&$ path $\% 5 \mathrm{~B} \% 5 \mathrm{D}=2549$

Barboza Jr., A. T. (2009). Ambientes Virtuais de Aprendizagem um estudo de caso no Ensino Fundamenta e Médio (Tesis inédita de maestría). Universidade Cruzeiro do Sul, Brasil.

Bittar, M. A. (2010). Escolha de um software educacional e a proposta pedagógica do professor: estudo de alguns exemplos da matemática. En: W. Beline; N.M. Lobo Da Costa. Educação Matemática, tecnologia e formação de professores: algumas reflexões (p. 215-242). Campo Mourão: Editora FECILCAM.

Borba, M. De C.; Silva, R. S. R. Da; Gadanidis, G. (2016). Fases das tecnologias digitais em educação matemática: sala de aula e internet em movimento. Belo Horizonte: Autêntica.

Caccuri, V. (2016). Tecnologia Digital para docentes: computación y TICs em el aula. Buenos Aires: Dalaga.

Dolz, J.; Schneuwly, B. (2004). Gêneros orais e escritos na escola. Campinas, Brasil: Mercado das Letras. Recuperado de https://archive-ouverte.unige.ch/unige:31458

Groenwald, C. L. O.; Zoch, L. N.; Homa, A. I. R. (2009). Sequência Didática com Análise Combinatória no Padrão SCORM. Bolema, Río Claro, 22(34), 27 - 55. Recuperado de https://www. redalyc.org/pdf/2912/291221876003.pdf

Homa, A. I. R.; Groenwald, C. L. O. (2016). Incluindo tecnologias no currículo de matemática: planejando aulas com o recurso dos tablets. Revista Unión, (48), 22-40. Recuperado de http://www.fisem.org/www/union/revistas/2016/48/132-674-1-ED_corrigido.pdf

Homa, A. I. R.; Groenwald, C. L. O. (2016). Área de figuras planas com objetos de aprendizagem no Geogebra. Revista Brasileira de Ensino de Ciência e Tecnologia, 9(1), 123-147. Recuperado de https://periodicos.utfpr.edu.br/rbect/ article/download/2000/2961
Homa, A. I. R. (2019). Robotics Simulators in STEM education. Acta Scientiae, 21(5), 178-191. Recuperado de https://www.researchgate.net/ publication/337264258_Robotics_Simulators in STEM education

Institute of Electrical and Electronics Engineers. (2002). IEEE Standard For Learning Object Metadata.

Kampff, A. J. C.; Machado, J. C.; Cavedini, P. (2004). Novas Tecnologias e Educação Matemática. Revista Novas Tecnologias na Educação, 2(2), 1-11. Recuperado de https://seer. ufrgs.br/renote/article/view/13703/16011

Kenski, V. M. (2012). Tecnologias e Ensino Presencial e a Distância. São Paulo, Brasil: Papirus.

Melo, K. M. F. (2013). A estatística nos anos finais do ensino fundamental: uma experiência com o tema transversal meio ambiente (Tesis inédita de maestría). Universidade Luterana do Brasil, Canoas, Brasil.

Merrill, D. (2002). Position statement and questions on learning objects research and practice. En Learning objects technology: Implications for educational research and practice, AERA, New Orleans, LA.

Ministério da Educação. Brasil. (1996). Lei de Diretrizes e Bases da Educação Nacional. Ministério da Educação.

Ministério da Educação. (2013). Guia de Tecnologias Educacionais da Educação Integral e Integrada e da Articulação da Escola com seu Território. Brasil: Secretaria de Educação Básica. Recuperado de http://portal.mec. gov.br/index.php?option=com_docman\&view $=$ download\&alias=14545-guia-tecnologias-20130923-pdf\&category_slug=novembro-2013-pdf\&Itemid=30192

Ministério da Educação. (2018). Base Nacional Comum Curricular. Brasil: Secretaria de Educação Básica. Recuperado de http://basenacionalcomum.mec.gov.br/images/BNCC EI_EF_110518_versaofinal_site.pdf

Mortimer, L. (2002). (Learning) objects of desire: promise and practicality. Learning Circuits. Recuperado de http://wwwq.learningcircuits. org/2002/apr2002/mortimer.html

NCTM. (2014). Principles to actions: ensuring mathematical sucess for all. Reston, VA: National Council of Teachers of Mathematics.

Nóvoa, A. (2007). Desafios do trabalho do professor no mundo contemporâneo. São Paulo: SINPRO. 
OCDE. (2010). Inspirados pela tecnologia, norteados pela pedagogia: uma abordagem sistêmica das inovações educacionais de base tecnológica. Florianópolis: CPEI. Recuperado de http://www.oecd.org/education/ ceri/47785311.pdf

Perrenoud, P. (2000). Dez novas competências para ensinar. Porto Alegre: Artes Médicas. Recuperado de https://abenfisio.com.br/wp-content/uploads/2016/06/10-novas-competencias-para-ensinar.pdf

Pietrovzki, P. (2015). Enseñar Matemática en la era digital. En: M. Libedinky; P. Pérez; X. G. Tellería, Las TIC em la Escuela Primaria. Buenos Aires: Centro de Publicaciones Educativas y Material didáctico.

Ramos, J. L.; Teodoro, V. D.; Ferreira, F. M. (2011). Recursos educativos digitais: reflexões sobre a prática. Cadernos SACAUSEF, 7, 11-34. Recuperado de http://dspace.uevora.pt/rdpc/ handle/10174/5051
Thomas, J.R.; Nelson, J. (2002). Métodos de pesquisa em atividade Física (3ra ed.) Porto Alegre: Artmed.

Valente, J. A. (2014). A comunicação e a educação baseada no uso das tecnologias digitais de informação e comunicação. Revista Unifeso Humanas e Sociais, 1(1), 141-166. Recuperado de http://unifeso.edu.br/revista/index. $\mathrm{php} /$ revistaunifesohumanasesociais/article/ download/17/24

Willey, D. (2002). The instructional use of learning objects. Bloomington, Indiana: Agency for Instructional Technology and Association for Educational Communications and Tecnology. Recuperado de https://members.aect.org/ publications/InstructionalUseofLearningObjects.pdf

Zabala, A. (1998). A prática educativa: como ensinar. Porto Alegre: Artmed.

\section{(c) $\underset{\mathrm{BY}}{\mathrm{NC}} \mathrm{\textrm {ND }}$}

As Tecnologias Digitais da Informação e Comunicação como um recurso didático no Currículo de Matemática (Iaqchan Ryokiti Homa-Agostinho y Claudia Lisete OliveiraGroenwald) in Uniciencia is protected by Attribution-NonCommercial-NoDerivs 3.0 Unported (CC BY-NC-ND 3.0) 